\title{
Severely atrophic human muscle fibers with nuclear misplacement survive many years of permanent denervation
}

\author{
Ugo Carraro (1), Helmut Kern (2,3) \\ (1) IRCCS, Fondazione Ospedale San Camillo, Venice, Italy; (2) Institute of Physical \\ Medicine and Rehabilitation, Wilhelminenspital, Vienna, Austria; (3) Ludwig Boltzmann \\ Institute of Electrical Stimulation and Physical Rehabilitation, Vienna, Austria
}

This article is distributed under the terms of the Creative Commons Attribution Noncommercial License (CC BY-NC 4.0) which permits any noncommercial use, distribution, and reproduction in any medium, provided the original author(s) and source are credited.

\begin{abstract}
Likewise in rodents, after complete spinal cord injury (SCI) the lower motor neuron (LMN) denervated human muscle fibers lose completely the myofibrillar apparatus and the coil distribution of myonuclei that are relocated in groups (nuclear clumps) in the center of severely atrophic muscle fibers. Up to two years of LMN denervation the muscle fibers with nuclear clumps are very seldom, but in this cohort of patients the severely atrophic muscle fibers are frequent in muscle biopsies harvested three to six years after SCI. Indeed, the percentage increased to $27 \pm 9 \%(\mathrm{p}<0.001)$, and then abruptly decreased from the 6th year onward, when fibrosis takes over to neurogenic muscle atrophy. Immunohistochemical analyses shown that nuclear misplacements occurred in both fast and slow muscle fibers. In conclusion, human muscle fibers survive permanent denervation much longer than generally accepted and relocation of nuclei is a general behavior in long term denervated muscle fibers.

Key Words: Human muscle, long-standing denervation, nuclear clumps
\end{abstract}

Eur J Transl Myol 2016; 26 (2): 76-80

\section{Nuclear positioning: Mechanisms and functional significance}

The nucleus is the largest organelle in the cells and its position is dynamically controlled in space and time. ${ }^{1}$ In the special case of adult skeletal muscle fibres of mammal muscles, there are hundreds or thousands of myonuclei that are typically distributed immediately under the plasmalemma and are spaced out along the fiber, except for a small cluster of specialized nuclei at the neuromuscular junction. ${ }^{2}$

During development and differentiation the nuclei migrate from the center of the new muscle fiber to the periphery and many are the genetic diseases that present centrally nucleated myofibers. All these aspects are explained in terms of events that interrupt the normal migration of the nuclei from the center to the periphery of the muscle fibers. ${ }^{3}$

The nuclear movements are mediated by the cytoskeleton which transmits pushing or pulling forces onto the nuclear envelope. While microtubules have been known for a long time to be key players in nuclear positioning, the actin and cytoplasmic intermediate filament cytoskeletons have been recently implicated in this functions. How the nuclei attain their positions along the fiber is not fully understood. Nuclei are preferentially localized near blood vessels (BV), particularly in slowtwitch, oxidative fibers. Thus, in rat soleus muscle fibers, $80 \%$ of the nuclei appear next to BV. Lack of desmin markedly perturbs the distribution of nuclei along the fibers but does not prevent their close association with BV. Consistent with a role for desmin in the spacing of nuclei, denervation affects the organization of desmin filaments as well as the distribution of nuclei. During chronic stimulation of denervated muscles, new BV form, along which muscle nuclei align themselves. The positioning of nuclei along muscle fibers is plastic and $\mathrm{BV}$ and desmin intermediate filaments each play a distinct role in control of this positioning. ${ }^{3}$

\section{Genetic Centronuclear Myopathy}

Centronuclear Myopathy (CNM) is a congenital myopathy characterized by prominence of central nuclei on muscle biopsy. CNM has been associated with mutations in MTM1, DNM2, and BIN1 but many cases remain genetically unresolved. RYR1 encodes the principal sarcoplasmic reticulum calcium release channel and has been implicated in various congenital myopathies. RYR1 mutations cause CNM. In addition to central nuclei, prominent histopathological findings included (often multiple) internalized nuclei and type 1 


\section{Human muscle fibers with nuclear misplacement}

Eur J Transl Myol 26 (2): 76-80

fiber predominance and hypotrophy with relative type 2 hypertrophy. Although cores were not typically seen on oxidative stains, electron microscopy revealed subtle abnormalities in most cases. These findings expand the range of RYR1-related phenotypes and suggest RYR1 mutations as a common cause of congenital myopathies with central nuclei. Corresponding to recent observations in X-linked CNM, these findings indicate disturbed assembly and/or malfunction of the excitationcontraction machinery as a key mechanism in CNM and related myopathies. ${ }^{3-5}$

\section{Denervation per se as a cause of nuclear misplacement}

On the other hand denervation per se is an important cause of nuclear misplacement. Indeed during permanent denervation in both rodents and humans up to the $90 \%$ of severely atrophic muscle fiber present a reorganization of the nuclear distribution. The permanent injury of lower motor neurons causes atrophy of skeletal muscle fibers that mainly occurs during the first few weeks/months. In rodents three to seven months after denervation, muscle fibers undergo a stage of slow progressive atrophy that result in a consistent reduction (up to 90\%) of the muscle tissue. At this late stage, the denervated muscle contains still numerous severely atrophic muscle fibers, some of which had lost all the contractile proteins and the coil distribution of myonuclei, which are aggregated in the center of the muscle fiber (nuclear clumps). At the same time, adipocytes and collagen sheets fill some of the empty spaces of the muscle and finally fibrosis prevails. ${ }^{6-8}$

In biopsies of quadriceps muscle harvested from human subjects who have experienced complete lower motor neuron (LMN) lesion (Conus and Cauda Syndrome) we observed similar events, but in a more extended period of time. ${ }^{9}$ Mild atrophy (that corresponds approximately to a decrease in muscle fiber size of the 50\%) appears in few weeks. It progresses during the first two years of denervation, while severe atrophy (a stage in which muscle fiber size decreases to $20-10 \%$ with respect to normal values) appears after two- three years, accompanied by a progressive degeneration of the muscle tissue. ${ }^{9,10}$ This behavior is not unique to humans, but it is a common feature of mammals larger than rodents. ${ }^{11,12}$

\section{Materials and Methods}

\section{Patients}

We collected muscle biopsies from quadriceps muscles of twenty-four patients who had experienced since one to ten years, traumatic spinal cord injury that caused complete lesion of the lumbar-ischiatic lower motor neurons (complete Conus and Cauda Syndrome). Demographic and clinical data of the patients are described in Kern H, et al. 2009. ${ }^{13}$ All subjects enrolled in the study were volunteers who received and signed a detailed informed consent. Clinical and functional assessments, as well as follow-up and muscle biopsies, were performed at the Wilhelminenspital, Vienna (Austria). Complete denervation of quadriceps muscle was assessed by electrophysiological testing, i.e. by test electrical stimulation, needle electromyography, brain motor control assessment, transcranial and lumbosacral magnetic stimulation. ${ }^{13}$ All applicable rules concerning the ethical use of human volunteers were followed during this study (Approval of Ethical Committee, Vienna, Austria: EK-02-068-0702).

\section{Hystological Analyses}

Light microscopy and morphometry of muscle biopsies were performed at the Interdepartmental Research Center of Myology, University of Padova (Italy), as described in Rossini et al. 2002. ${ }^{14}$ In brief, after either freezing or fixing and embedding the specimens, cryosections $(10 \mu \mathrm{m}$ thickened) or thin section $(1 \mu \mathrm{m}$ thickened), were collected on glass slides and stained with hematoxylin-eosin (H\&E) or toluidine blue, respectively, using conventional techniques. All images were collected using a Zeiss microscope connected to a Leica DC 300F camera under the same conditions that were used to acquire a reference ruler. Data were expressed as mean \pm SD. To detect significant differences between groups, we performed the two-tailed Student's t-test for unpaired observations. The significance level was set to $\mathrm{p}=$ 0.05 .

\section{Immunohistochemical Analyses}

Unfixed muscle sections were labeled for 1 hour at room temperature using mouse monoclonal antibody directed against slow-type myosin heavy chain (MHCs) (Novocastra, Newcastle-upon-Tyne, U.K), 1:20 diluted in Tris-buffered saline (TBS). Sections were rinsed $3 \times 5 \mathrm{~min}$ in TBS, and then incubated for 1 hour at room temperature with FITC labeled conjugates directed against mouse IgG (SigmaAldrich, St. Louis, USA) 1:200 diluted in 10\% goat serum/TBS. Negative controls were performed by omitting the primary antibodies on samples. After washes, nuclei were counterstained for $5 \mathrm{~min}$ at room temperature with Hoechst 33258 (Sigma-Aldrich, St. Louis, USA), sections were coverslipped using mounting medium (Dako, Glostrup, Denmark) and observed under a Zeiss microscope. ${ }^{14}$

\section{Severely atrophic muscle fibers with nuclear clumps}

Figure 1 show H\&E stained cross sections of skeletal muscle biopsies harvested from normal (A) and denervated subjects at 0,8 (B), and 4,1 (C) years after spinal cord injury. Normal muscle (A) is characterized by well-packed myofibers of 58,3 $\pm 11,6$ mean fiber diameter $(\mu \mathrm{m} \pm \mathrm{SD})$. Minimal interstitial connective tissue is present. One year after complete lower motor neuron lesion (B), the muscle is still characterized by 


\section{Human muscle fibers with nuclear misplacement}

Eur J Transl Myol 26 (2): 76-80

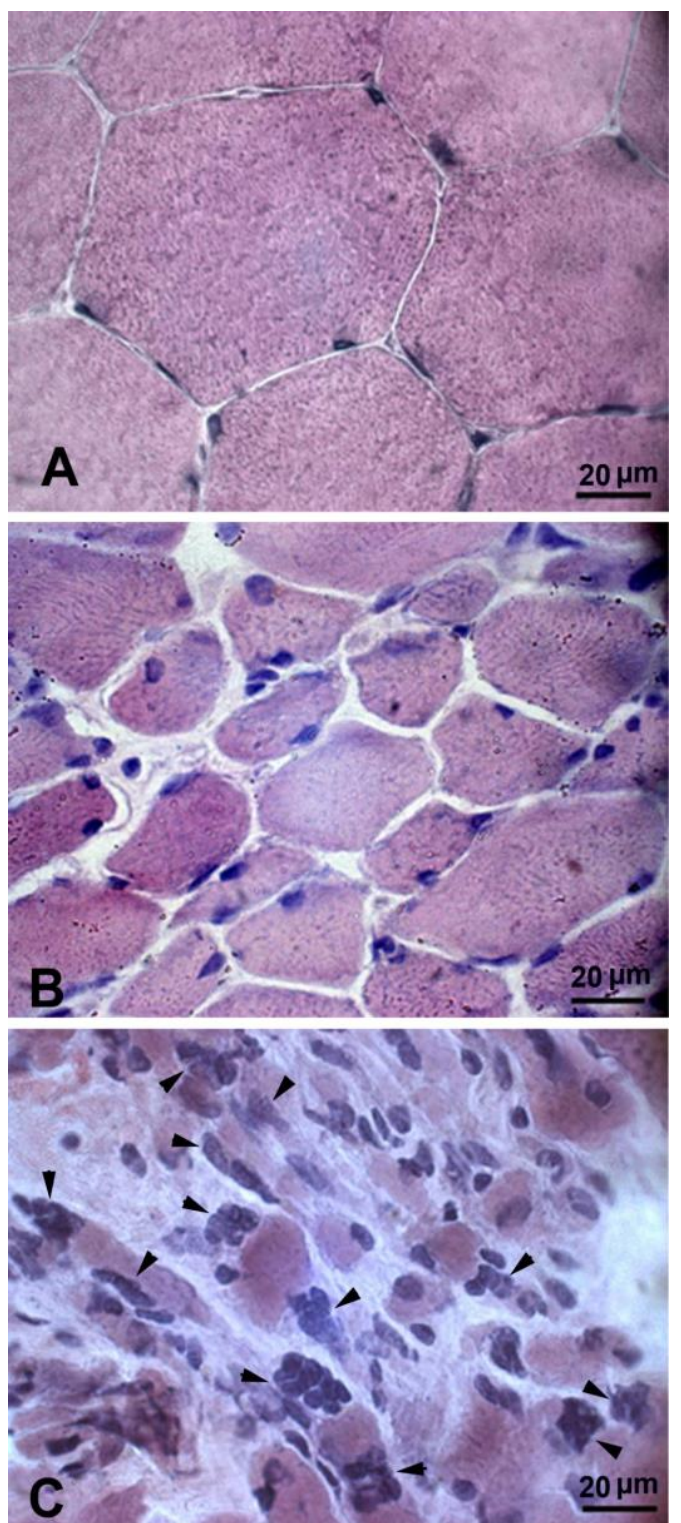

Fig 1. Severely atrophic muscle fibers with nuclear clumps survive many years in permanently denervated human muscle. Normal and LMN denervated quadriceps muscle. Scale bar: 20 um. H\&E stain. (A) Normal muscle. (B) 0,8 year denervated muscle characterized by mild atrophic myofibers, with connective tissue of almost normal appareance. (C) 4,1 year denervated muscle showing severely atrophic myofibers in which the contractile apparatus is almost absent and three or more clumped nuclei (arrowheads) can be identified. Reprinted from The Open Pathology Journal, 2009, 3, 106-110. ${ }^{24}$

variable mild atrophy with myofibers of $30,2 \pm 10,8$ mean fiber diameter $(\mu \mathrm{m} \pm \mathrm{SD})$. Noteworthy, nuclei are still peripherally located.

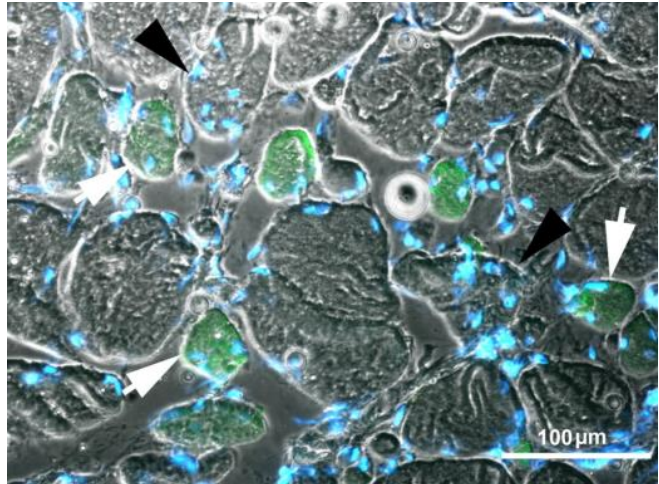

Fig 2. Immunohistochemical staining with antiMHCs shows that both fast (larger, not stained muscle fibers pointed by black arrowheads) and the green-labeled slow type muscle fibers (white arrows) present several central nuclei. Scale bar: $100 \mu \mathrm{m}$. Reprinted from The Open Pathology Journal, 2009, 3, 106-110. ${ }^{24}$

After four years from lower motor neuron injury (C), muscle biopsy is characterized by numerous very atrophic fibers $(<15 \mu \mathrm{m}$ mean fiber diameter) separated by increased fibrous tissue. Arrowheads point to the severely atrophic myofibers in which the contractile apparatus seems to be almost absent and five or more nuclei fill the cross section of some of them (nuclear clumps).

In muscle sections from biopsies harvested from one to two years after complete denervation $(n=11)$, we observed $2 \pm 5 \%$ of myonuclear clumps (mean \pm SD). In biopsies harvested three to five years after spinal cord injury $(\mathrm{n}=7)$, the percentage abruptly and significantly increased to $27 \pm 9 \%(\mathrm{p}<0,001)$. The value significantly decreased in biopsies $(n=6)$ harvested more than six years from spinal cord injury to $4 \pm 4 \%$ ( $p<0,001$ vs three-six years of denervation). Figure 2 shows a cryosection from a 5.4-year LMN denervated human muscle biopsy after staining in green with an anti-MHCs antibody. The white arrows point to slow-type green atrophic muscle fibers with centralized nuclei, labeled in blue by Hoechst 33258 . The black arrowheads indicates two less atrophic fast type muscle fibers (not stained by the anti-MHCs antibody), which contains several centralized nuclei. These morphological aspects suggest that the severe atrophy with nuclear redistribution is the results of the progressive disorganization of the sarcomeric stuctures of the denervated muscle fibers, which meantime lose the normal coil distribution of myonuclei. Whatever the mechanisms of their rearrangement, the muscle nuclei are for many months (or years) grouped in clusters of tens of nuclei that fill the severely atrophic muscle fibers, being separated by long stretches of amyofibrillar myoplasm. 


\section{Human muscle fibers with nuclear misplacement}

Eur J Transl Myol 26 (2): 76-80

In biopsies harvested from patients affected with complete Conus and Cauda syndrome (that is, complete and permanent lower motor neuron lesion at lumbar-ischiatic level of spinal cord), the muscle tissue, before undergoing adipose and fibrous tissue substitution, progressively shows a severe atrophy of muscle fibers that loses their myofibrillar apparatus and relocates in clumps their nuclei. ${ }^{9}$ These features are in sharp contrast with the stable muscle atrophy we described in long-term paraplegics with complete upper motor neuron lesion from three to twenty years of spinal cord injury at thoracic level. ${ }^{15}$ Interestingly, we never observed nuclear clumps in that type of disuse atrophy. Up to now, nuclear groupings have been described in muscle biopsies from patients affected with neurodegenerative disorders, like amyotrophic later sclerosis and spinal muscular atrophy. ${ }^{16,17}$ In those cases, nuclear clumps are rarely present in muscle fibers empty of their sarcomeric structures, because in neurodegenerative diseases the incompleteness of denervation allows reinnervation and thus the reorganization of larger muscle units. ${ }^{17}$

Our observations thus confirm that human muscle fibers survive complete and permanent denervation much longer than generally accepted. Nuclear clumps should be considered markers of the long-lasting ability of mammals muscle fibers to survive to the absence of the nerve. ${ }^{18}$ These results provide the rationale to plan research aimed to recover these severely atrophic myofibers, by combining molecular and cellular approaches with functional electrical stimulation that our previous studies shown to restore muscle structure and mass in human long-term denervated and degenerated muscle. $^{19-24}$

\section{Acknowledgement}

U.C. thanks the Interdepartmental Research Center of Myology at the Department of Biomedical Sciences, University of Padova, Italy for hospitality. The research was supported by the EU Commission Shared Cost Project RISE (Contract no. QLG5-CT-2001- 02191), and by Ludwig Boltzmann Institute of Electrostimulation and Physical Rehabilitation funds, Vienna, Austria.

\section{Conflict of Interest}

The author declare no conflict of interests.

\section{Corresponding Author}

Ugo Carraro, I.R.C.C.S. Fondazione Ospedale San Camillo. Via Alberoni, 70 - 30126 Venezia-Lido, Italy Phone: +39338 1575745

E-mail: ugo.carraro@ospedalesancamillo.net

\section{References}

1. Dupin I, Etienne-Manneville S. Nuclear positioning: mechanisms and functions. Int $\mathrm{J}$ Biochem Cell Biol 2011;43:1698-707. doi: 10.1016/j.biocel.2011.09.004. Epub 2011 Sep 21. Review.
2. Ralston E, Lu Z, Biscocho N, et al. Blood vessels and desmin control the positioning of nuclei in skeletal muscle fibers. J Cell Physiol 2006;209:874-82.

3. Wilmshurst JM, Lillis S, Zhou H, et al. RYR1 mutations are a common cause of congenital myopathies with central nuclei. Ann Neurol 2010;68:717-26. doi: 10.1002/ana.22119.

4. Galli L, Orrico A, Lorenzini S, et al. Frequency and localization of mutations in the 106 exons of the RYR1 gene in 50 individuals with malignant hyperthermia. Hum Mutat 2006; 27:830.

5. Paolini C, Quarta M, Wei-LaPierre L, et al. Oxidative stress, mitochondrial damage, and cores in muscle from calsequestrin-1 knockout mice. Skelet Muscle 2015;5:10. doi: 10.1186/s13395-015-0035-9. eCollection 2015.

6. Adami N, Kern H, Mayr W, et al. Permanent denervation of rat Tibialis Anterior after bilateral sciatectomy: Determination of chronaxie by surface electrode stimulation during progression of atrophy up to one year. Basic Appl Myol 2007; 17:237-43.

7. Borisov AB, Dedkov EI, Carlson BM. Interrelations of myogenic response, progressive atrophy of muscle fibres, and cell death in denervated skeletal muscle. Anat Rec 2001;264:203-18.

8. Carlson BM. "The Denervated Muscle" - 45 years later. Basic Appl Myol 2007;17:113-7.

9. Kern H, Boncompagni S, Rossini K, et al. Longterm denervation in humans causes degeneration of both contractile and excitation- contraction coupling apparatus, wich is reversibile by functional electrical stimulation (FES). A role for myofiber regeneration? J Neuropathol Exp Neurol 2004;63:919-31.

10. Biral D, Kern H, Adami N, et al. Atrophyresistant fibers in permanent peripheral denervation of human skeletal muscle. Neurol Res 2008;30:137-44.

11. Ashley Z, Sutherland H, Lanmuller H, et al. Atrophy, but not necrosis, in rabbit skeletal muscle denervated for periods up to one year. Am J Cell Physiol 2007;292:440-51.

12. Lewis DM, Al-Amood WS, Schmalbruch H. Effects of long-term phasic electrical stimulation on denervated soleus muscle: guinea pig contrasted with rat. J Muscle Res Cell Motil 1997;18:573-86.

13. Kern H, Hofer C, Mayr W, Carraro U. European Project RISE: Partners, protocols, demography. Basic Appl Myol/Eur J Transl Mycol 2009;19:211-6.

14. Rossini K, Zanin ME, Podhorska-Okolow M, Carraro U. To stage and quantify regenerative myogenesis in human long-term permanent 


\section{Human muscle fibers with nuclear misplacement}

Eur J Transl Myol 26 (2): 76-80

denervated muscle. Basic Appl Myol 2002;12:27786.

15. Kern H, Hofer C, Modlin M, et al. Stable muscle atrophy in long term paraplegics with complete upper motor neuron lesion from 3- to 20-year SCI. Spinal Cord 2008;46:293-304.

16. Dubowitz V, Sewry CA. Muscle biopsy. A practical approach. Sauders Elsevier Edition 2007. Neurol 2004;63:919-31.

17. Wokke JH. Genes, trials, and care: The dynamics of neuromuscular disease. Lancet Neurol 2004;3:116.

18. Squecco R, Carraro U, Kern H, et al. A subpopulation of rat muscle fibers maintains an assessable excitation-contraction coupling mechanism after long-standing denervation despite lost contractility. J Neuropathol Exp Neurol. 2009;68:1256-68. doi: 10.1097/NEN. 0b013e3181c18416.

19. Boncompagni S, Kern H, Rossini K, et al. Structural differentiation of skeletal muscle fibers in the absence of innervation in humans. Proc Natl Acad Sci USA 2007; 104: 19339-44.
20. Kern H, Salmons S, Mayr W, Rossini K, Carraro $\mathrm{U}$. Recovery of long-term denervated human muscles induced by electrical stimulation. Muscle Nerve 2005; 31: 98-101.

21. Kern H, Carraro U. Translational myology focus on clinical Challenges of functional electrical stimulation of denervated muscle. Basic Appl Myol/Eur J Transl Myol 2008; 18: 37-100.

22. Kern H, Carraro U, Adami N, et al. One year of home-based Functional Electrical Stimulation (FES) in complete lower motor neuron paraplegia: Recovery of tetanic contractility drives the structural improvements of denervated muscle. Neurol Res 2009, in press.

23. Kern H, Hofer C, Mayr W. Protocols for clinical work package of the European project RISE. Basic Appl Myol/Eur J Transl Myol 2008; 18: 3944.

24. Kern H, Carraro U, Biral D, Adami N, Zampieri S. Severely atrophic muscle fibers with nuclear clumps survive many years in permanently denervated human muscle. The Open Pathology Journal 2009 Aug 31;3(1). 